\title{
Development of an automated bond verification system for advanced electronic packages
}

\author{
Michel Darboux, Jean-Marc Dinten \\ LETI (CEA - Technologies Avancées) - DSYS/SCSI \\ CENG, 17 rue des Martyrs \\ F38054 Grenoble Cedex 9 - FRANCE
}

\begin{abstract}
As the performance of an electronic product improves, the surface density of the board population increases drastically. As a consequence, the technology is moving from conventional components to surface mounted components. The solder joints are more and more difficult to inspect, they are no longer reachable by the standard inspection techniques (visible light or laser). However, X-rays still allow to access information concerning these joints. In this paper, we propose a diagnosis method for characterising solder joints of surface mounted components. This method is based on a comparison between three radiographic images acquired from a mounted component, the corresponding unsoldered component used as a model, and the unpopulated board. Firstly, the radiographies are preprocessed in order to get quantitative information. Then, an angle and $\mathrm{X}, \mathrm{Y}$ position alignment is performed before the subtraction of the unpopulated board and the model component images from the mounted component image. This leads to the solder joint map, from which quantitative parameters are then extracted. After a description of the image processing algorithms, we present experimental results obtained on a typical component. These results show clearly the possibility to access important parameters characterising the solder joint.
\end{abstract}

\section{Introduction}

Compared to visible light or laser, $\mathrm{X}$-rays provide additional information. Radiographies show the internal structures while visible or laser images describe the surface. This property is essential for printed circuit board inspection. X-rays allows the access to information unreachable by other inspection techniques : internal defects, solder hidden by a component (as for BGA or J-lead components for example).

For a complex object as an electronic board, the radiography shows several 3D structures that are superimposed; therefore, direct interpretation is not obvious. Standard ways to deal with this superimposition problem are tomography and laminography. Tomography leads to a 3-D reconstruction of the lead and the solder, but requires a large number of radiographies and powerful computers. This is not possible for "on-line" industrial inspection. Laminography allows to

\footnotetext{
* This work has been achieved within the BRITE-EURAM project "VERBONDS".
} 
reconstruct rapidly a "blurred" image of one slice of the object. It is well suited for detecting defects like a lack or an excess of solder, but it is not adapted for a quantitative characterisation of the solder joint. At LETI, an approach taking into account this superimposition has been developed [1]. This method allows the separation of the contribution of each side of a double sided mounted board, by combining three radiographies taken from different points of view.

Once the separation of the data from each side of the board is achieved, the respective contributions of the solder joint, the lead and the traces, must be identified. To do this, a diagnosis method for solder joint analysis is proposed. It is based on image comparison using a model of the object (the unsoldered electronic component). This approach enables to focus on defect detection and quantitative parameters extraction.

\section{General presentation}

The proposed X-ray inspection method of printed circuit boards solder joints is based on image comparison. The main steps of this method are presented in figure 1. We use three radiographies : one field from the inspected mounted board, the corresponding field from the unpopulated board, the radiography of the model component. The first step is a preprocessing of each radiography in order to obtain quantitative information. The second step is a spatial alignment (in angle and X,Y position) in order to be able to subtract the unpopulated board and the model component images from the mounted board image. The combination of these three radiographies leads to the solder joint map from which quantitative parameters are then extracted.

For on-line inspection applications, the model components radiographies could be taken from a database previously generated.

\section{Preprocessing of the radiographic images}

The initial image from the acquisition system shows the remaining flux of X-ray photons after crossing the object to be inspected (see section 5). The initial image pixels have integer values (encoded between 0 and 4095). The purpose of this preprocessing is to transform the pixel values of this initial image into thickness information. To correct the bias introduced by the acquisition system, we use a reference image obtained with a uniform object and a black image obtained when the X-ray tube is off. In the resulting image, the pixel real value is the product of the thickness by the corresponding linear attenuation coefficient, for the different layers composing the printed circuit board.

\section{The spatial alignment}

\subsection{X,Y position alignment}

A binary correlation technique is used. The alignment is done for the mounted board and the model component images and then for the mounted board and 
the unpopulated board ones. It consists first of a gradient computation on the two images to be aligned. The grey levels gradients are then binarized, using a threshold calculated as a percentage of a maximum gradient value. This value is determined after removing the possible false maximum due to noisy information. The correlation function is then computed between the two binarized images. The coordinates of the maximum of the function give the $\mathrm{X}$ and $\mathrm{Y}$ misalignment.

\subsection{Angle alignment}

To make a good comparison, it is necessary to have the same orientation for the mounted component, the model component, and the unpopulated board. This is obtained by correcting the orientation of each image. The reference orientation is a vertical line. The correction angle to apply is shown on the right. The angle alignment algorithm is now detailed. Firstly, the gradient of the image is extracted. These grey level gradient values are then projected along the columns, and the standard deviation of the projected signal is measured. The image is rotated for different angles, the misalignment angle is obtained for a rotation that gives the maximum standard deviation. The example given in figure 2 shows how the standard deviation of the projected signal decreases when the image is not correctly aligned along the vertical reference.

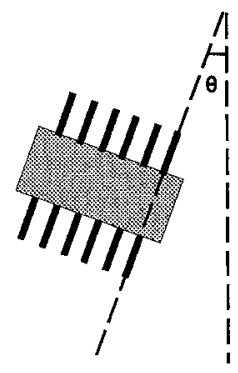

correction angle

\section{Experimental conditions}

The system used for the radiographic acquisitions is now described (see the figure below). The X-ray source is a continuous emitting microfocus tube with a minimum focus spot size of 10 microns, and a power range from 30 to 160 $\mathrm{KV}$. The inspected board is placed between the microfocus tube and an X-ray image intensifier. The visible light image at the output of the intensifier is then acquired with a CCD camera (512x512 pixels). The resolution depends on the position of the sample between the tube and the intensifier. Concerning the objects to be inspected, an industrial printed circuit board with various SMT (Surface Mount Technology) components is used. The results obtained on a typical J-lead component are presented hereafter.

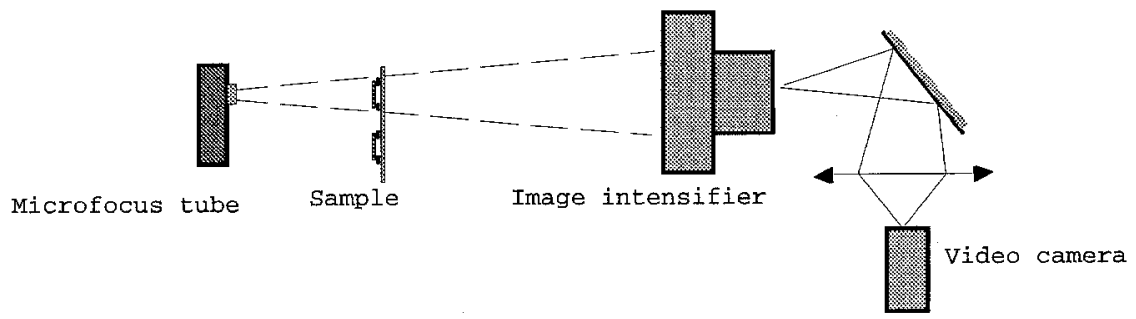




\section{Experimental results}

A schematic representation of a J-lead component and solder joint is given on the right. Figure 3 shows the images obtained with the microfocus acquisition system for a $\mathrm{J}$-lead component (the pixel size is 17.4 microns for the $\mathrm{X}$ axis and 12.3 microns for the $\mathrm{Y}$ axis), and the cross sections of one of the solder joints (for these graphical representations, the size of the grid is 100 microns by 100 microns). We can see on these graphs the different parameters characterising the solder joint. The legend for these parameters is, $\theta$ : wetting angle, $\mathrm{L}:$ joint length, $\mathrm{W}$ : joint width, $\mathrm{H}$ : heel fillet height. We can note for this

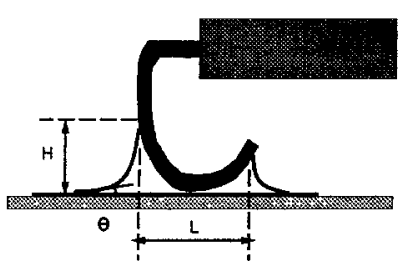

J-lead solder joint example, that this system allows to obtain reliable parameters. These parameters fit well with the real physical shape of the J-lead joint obtained with the scanning electron microscope.

\section{Conclusion}

The experimental results presented in this paper show clearly the possibility to access important parameters characterising solder joints. Many of these parameters are unreachable by visible or laser inspection techniques.

These parameters are well correlated with the real physical shape of the joints obtained with the scanning electron microscope.

All the algorithms presented have been developed under an interactive image processing environment. At present time their use is highly supervised. The next step would be to automate the processing : the user would just have to define a region of interest on the mounted board and the system would then automatically execute the combination and provide a list of relevant parameters.

The model component may present some differences with the mounted component (tolerances of fabrication and acquisition). LETI is working on the development of methods integrating these kinds of tolerances in the analysis [2].

\section{References}

[1] C. Icord, P. Rizo and JM. Dinten. X-ray quantitative evaluation of multilayered objects, QNDE94, SNOWMASS, CO, USA, August 1994.

[2] S. Girard, JM. Dinten and B. Chalmond. Automatic building of radiographic flexible models using a set of examples, Fifth International Conference on Image Processing and Its Applications, Edinburgh, UK, July 1995. 


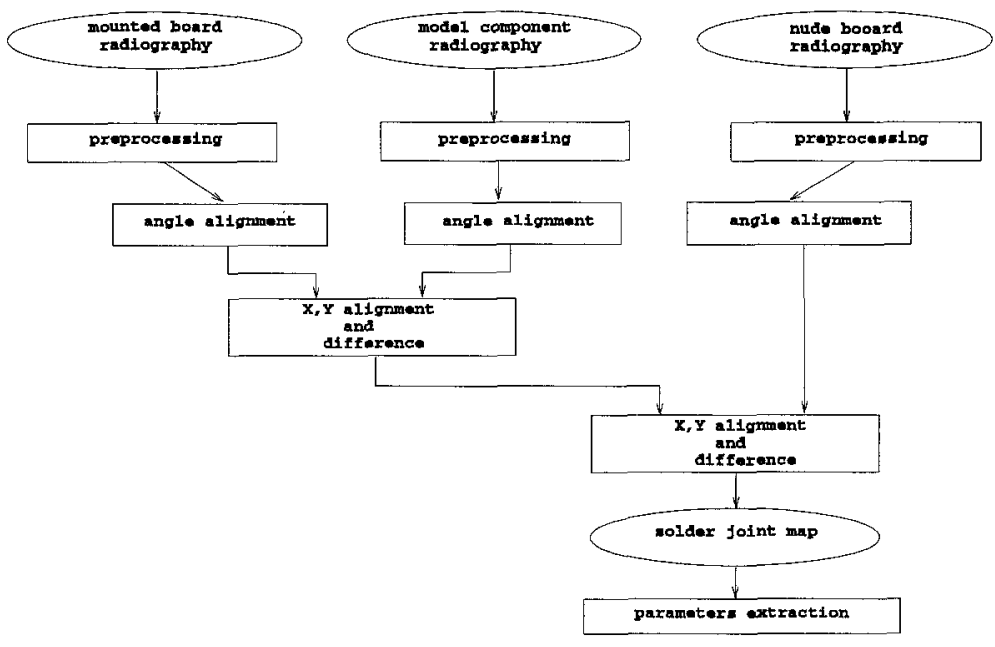

Fig. 1. the main steps of the inspection method

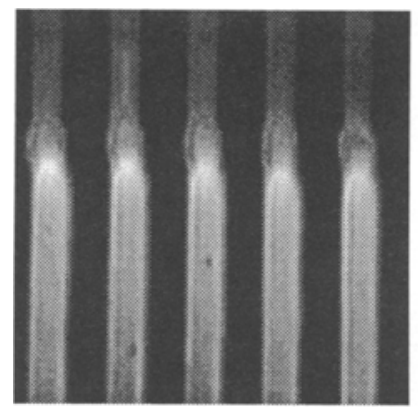

initial image

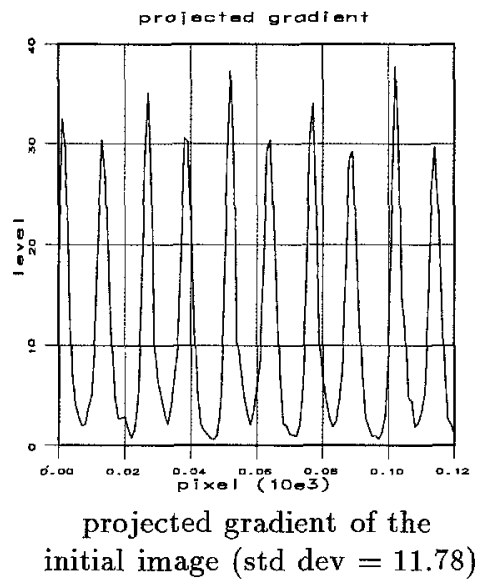

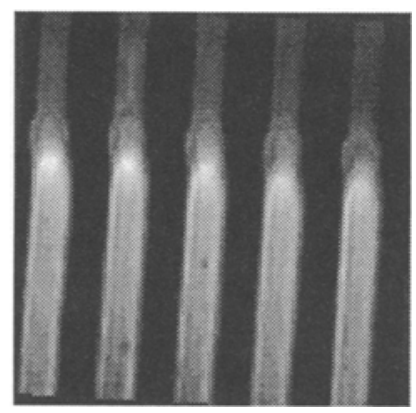

rotated image (3 degrees)

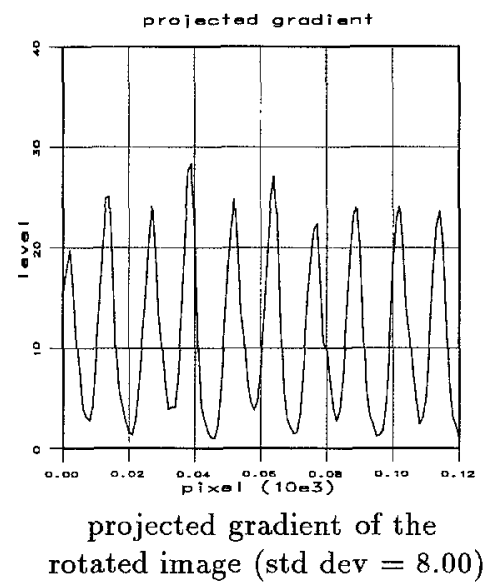

Fig. 2. angle alignment principle 


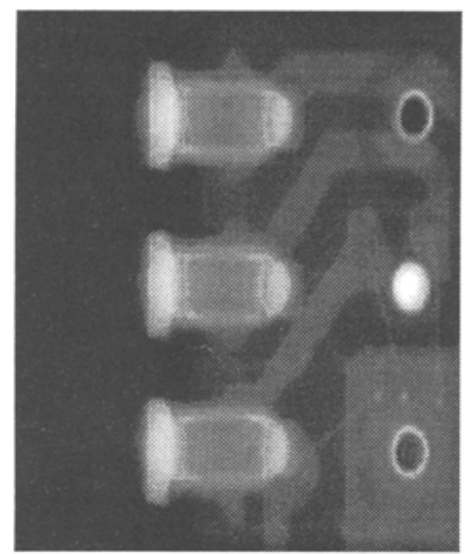

mounted board

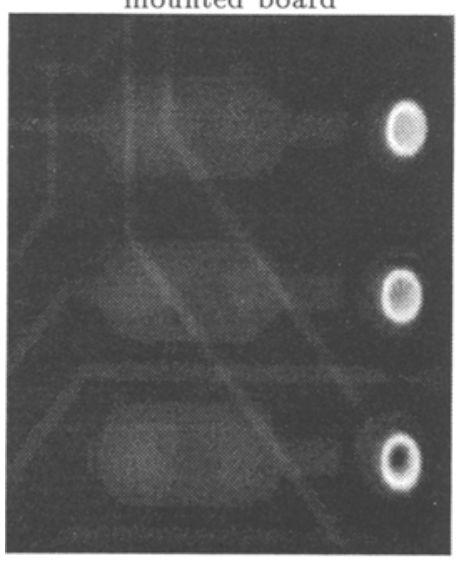

unpopulated board

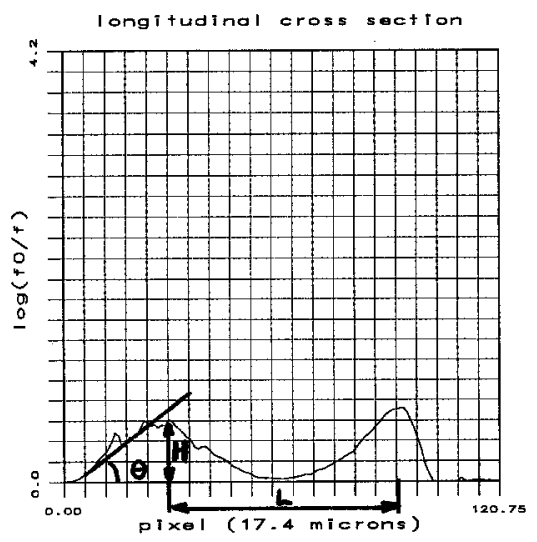

longitudinal cross section

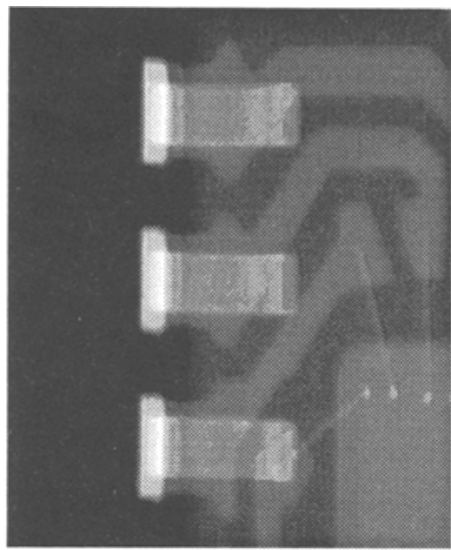

model component

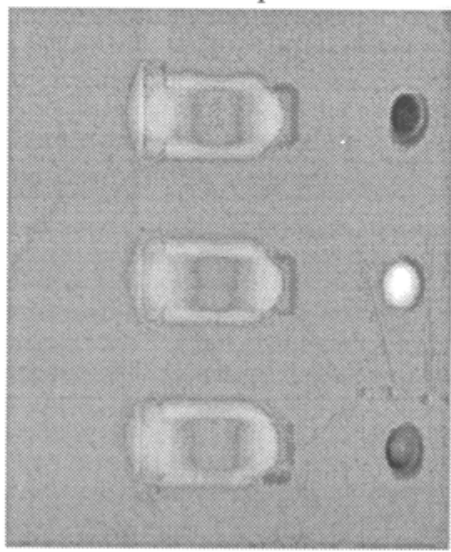

solder map

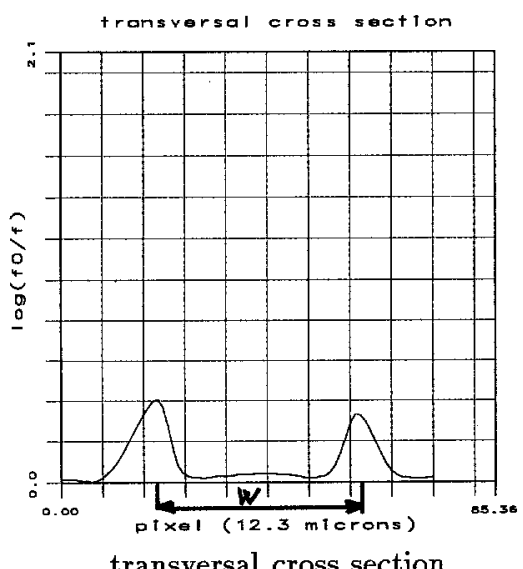

transversal cross section

Fig. 3. J-lead component subimages and solder joint cross sections 